\title{
Some Remarks on Design of LED Lamps and their AC Direct Drivers
}

\author{
Zdzislaw Filus, Jacek Checinski \\ Institute of Electronics, Silesian University of Technology, \\ Akademicka St.16, 44-100 Gliwice, Poland \\ zdzislaw.filus@polsl.pl
}

\begin{abstract}
Many examples of capacitorless and inductorless drivers used to power LED lamps directly from the AC mains use complicated circuitry in the form of a dedicated integrated circuit which is designed for a particular multistring lamp in order to obtain the value of the power factor very close to 1 and high efficiency of the whole unit. However, the authors of such descriptions do not provide any details concerning the optimum choice of the number of strings in a LED lamp and the number of LEDs in each string. The aim of this work is to show that acceptable values of both electrical parameters mentioned above can be obtained using a LED lamp with just one or two strings. Some formulae and results of their numerical analysis for the $230 \mathrm{~V} 50 \mathrm{~Hz}$ mains are presented, thanks to which designers of LED lamps and their AC direct drivers may easily estimate the optimum conduction voltage of a LED string or the number of single LEDs in a string.
\end{abstract}

Index Terms-Driver circuit; Energy efficiency; LED lamp.

\section{INTRODUCTION}

Solid-state lighting, based on light emitting diodes (LED), has been rapidly replacing conventional sources of light such as incandescent bulbs or compact fluorescent lamps (CFL). The main advantages of LED lamps are high luminous efficiency, long lifetime and ease of changing both the colour and intensity of the produced light. However, whereas incandescent bulbs can be directly connected to the AC supply network and CFLs require only some simple circuitry to periodically ignite gas discharge in the lamp, feeding of LED lamps is much more complicated. As they generate light when they are forward biased and their current versus voltage characteristics are steep, they require feeding with a constant, or periodically constant current - like in the case of pulse width modulated (PWM) waveform. If LEDs were fed from voltage sources, a small variation of voltage would cause much greater change in current. This could result in a reasonable change of the LED's operating temperature and, consequently, change of the light intensity and chromatic parameters. A serious drawback of LEDs in lighting applications is their fast response to the supply current change; when the current drops to zero, the lamp almost immediately goes out. The above mentioned problems are especially difficult to solve when LED lamps are supplied

Manuscript received 8 November, 2018; accepted 28 January, 2019.

This research was performed within statutory activities supported by the Polish Ministry of Science and Higher Education. from the AC mains. Incandescent bulbs, on the contrary, are very well suited for this type of supply because they produce light thanks to thermal effects, so flickering is negligible. They have been withdrawn from common use only because of their low efficiency.

\section{AC DRIVERS FOR LED LAMPS}

In most applications LED lamps should preferably be supplied from the AC mains. As a forward voltage drop for a single LED is just a few volts, they are connected in series into LED strings with the conduction voltage of at least tens of volts. To force a constant current flow through an LED string, or to produce a PWM waveform, many designs of drivers are used, whose thorough overview is included in [1]. LED drivers are divided, in general, into the passive ones and switched-mode drivers. In passive drivers a stepdown transformer is used to reduce the line voltage and the secondary voltage is then rectified and filtered with an electrolytic capacitor. The voltage across the capacitor feeds the LEDs through a current controlling resistor. To reduce the line voltage, a series inductor or a capacitor is sometimes used instead of the transformer. In switched-mode drivers the line voltage is first rectified and then reduced to the required value by a DC/DC converter. In both approaches a sufficiently large electrolytic capacitor is used in order to greatly reduce flicker of the LED lamp at a frequency of $100 \mathrm{~Hz}$. Such flicker considerably deteriorates the quality of light and in some applications may be even dangerous due to the stroboscopic effect. Capacitive load results in low values of the power factor, so power factor correction circuits (PFC) are often used with the DC/DC converters, further complicating their structure. LED lamps are usually designed to fit into a typical bulb socket and this means that the whole driver must be integrated into the lamp housing. As the lifetime of electrolytic capacitors is much shorter than that of LEDs, this greatly reduces the lifetime of the whole unit. To overcome this difficulty, another solution has recently become an interesting alternative in which electrolytic capacitors, as well as bulky inductive components, are eliminated [2]-[8].

\section{AC DIRECT LED DRIVERS}

The idea of such drivers is shown in Fig. 1. The lamp consists of a number of LED strings that are sequentially 
turned on, depending on the instantaneous voltage $V_{I N}$ from the rectifier. When this voltage reaches $V_{L E D I}$, the switch $\mathrm{S}_{1}$ closes and the current starts flowing through the first string. When $V_{I N}$ increases to $V_{L E D 1}+V_{L E D 2}, \mathrm{~S}_{2}$ closes and $\mathrm{S}_{1}$ opens, so the current $I_{2}$ starts flowing through LED1 and LED2 connected in series. The process continues until $\mathrm{S}_{\mathrm{n}}$ is closed. With $V_{I N}$ decreasing the switches are turned over in the reverse order. The currents $I_{1} \ldots I_{n}$ may be either the same [2] or they may change to make the staircase supply current waveform resemble a sine wave (Fig. 2) [3]-[6]. They may even consist of sections of a sine wave [7] in order to maximize the power factor $(P F)$ of the driver.

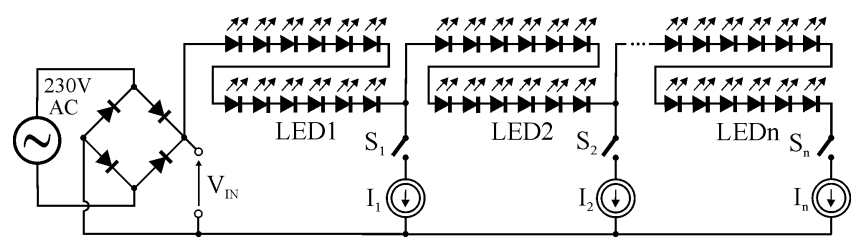

Fig. 1. Simplified circuit diagram of AC direct LED drivers.

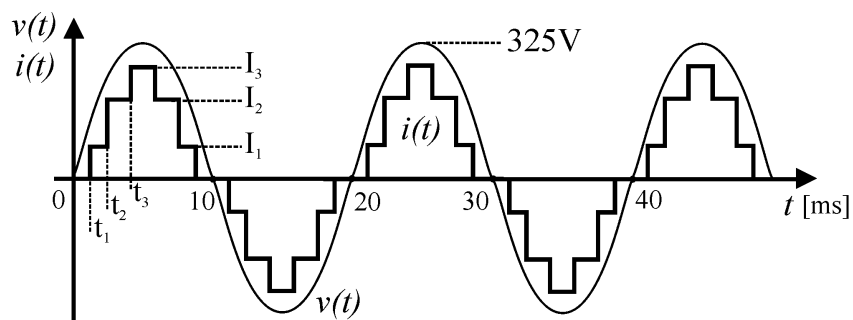

Fig. 2. Typical waveforms of the supply voltage and current in AC direct LED drivers.

Except for the rectifier, the driver is usually designed as a dedicated integrated circuit [2]-[6], [8] and the parameters of the circuit are often obtained from simulations [2], [6], [7]. The authors of the cited references claim to have achieved the power factor of their designs exceeding 0.99 and the efficiency of about $85 \%-90 \%$. However, $P F$ almost reaching the ideal value, that is 1 , is not necessary. According, for example, to the ENERGY STAR (USA) requirements [9] the following conditions should only be met: $P F \geq 0.6$ for LED lamps with the rated power from the range $5 \mathrm{~W}-10 \mathrm{~W}$ and $P F \geq 0.7$ for higher power values. European regulations set the following limits [10]: $P F \geq 0.4$ for $2 \mathrm{~W}<P \leq 5 \mathrm{~W}, P F \geq 0.5$ for $5 \mathrm{~W}<P \leq 25 \mathrm{~W}$ and $P F \geq 0.9$ for $P>25 \mathrm{~W}$.

What is more, in the published descriptions of drivers no criteria or hints are described concerning the optimum choice of the LED string conduction voltage (number of LEDs in a string) or the number of strings, which is usually taken arbitrarily to be, say, 5-10. The conduction voltage of a single string is usually not stated.

The aim of this work is to show that by a proper choice of the conduction voltage of a string, acceptable values of the power factor and efficiency of a lamp with only one or two strings can be achieved.

\section{INFLUENCE OF LED STRING VOLTAGE ON POWER FACTOR AND EFFICIENCY}

For simplicity, in further analyses we will ignore dynamic resistance of LEDs and thermal effects, voltage drops across rectifier diodes and assume that at a moment when a string starts conducting, the voltage across a relevant current source is zero. This will lead to substantial errors of the results for low voltages of LED strings. However, in lamps fed from the mains the conduction voltage of a single string is at least a few tens of volts. What is more, high accuracy of analysis is not necessary because the choice of the conduction voltage of a string for an optimized lamp will only be possible by steps equal to the conduction voltage of a single LED, which is about $3 \mathrm{~V}$.

If all currents $I_{l} \ldots I_{n}$ (Fig. 2) are the same and equal $I$, the power factor of the driver is independent of the number of LED strings and becomes

$$
\begin{gathered}
P F=\frac{P_{A C}}{V_{R M S} I_{R M S}}=\frac{\frac{4}{T} \int_{t_{1}}^{\frac{T}{4}} I V_{m} \sin \frac{2 \pi}{T} t d t}{\frac{V_{m}}{\sqrt{2}} \sqrt{\frac{4}{T} \int_{t_{1}}^{\frac{T}{4}} I^{2} d t}}= \\
=\frac{2 \sqrt{2} \cos \frac{2 \pi}{T} t_{1}}{\pi \sqrt{1-4 \frac{t_{1}}{T}}},
\end{gathered}
$$

where $P_{A C}$ is the active power drawn from the mains, $V_{R M S}$ and $I_{R M S}$ are the supply RMS voltage and current, $V_{m}$ and $T$ are the amplitude and the period of the mains voltage, respectively, and $t_{l}$ is the moment of turning on the first LED string (Fig. 2). We integrate over a quarter of the period only as the integrals in the remaining three quarters have the same values.

The efficiency $\eta$ of the driver with a lamp including one string only is given by the following equation

$$
\begin{gathered}
\eta=\frac{P_{L E D}}{P_{A C}}=\frac{\frac{4}{T} \int_{t_{1}}^{\frac{T}{4}} I V_{m} \sin \frac{2 \pi}{T} t_{1} d t}{\frac{4}{T} \int_{t_{1}}^{\frac{T}{4}} I V_{m} \sin \frac{2 \pi}{T} t d t}= \\
=2 \pi\left(\frac{1}{4}-\frac{t_{1}}{T}\right) \tan \frac{2 \pi}{T} t_{1}
\end{gathered}
$$

where $P_{L E D}$ is the power drawn solely by the LED string.

Both relationships, calculated for the $230 \mathrm{~V} 50 \mathrm{~Hz}$ line voltage, are shown graphically in Fig. 3.

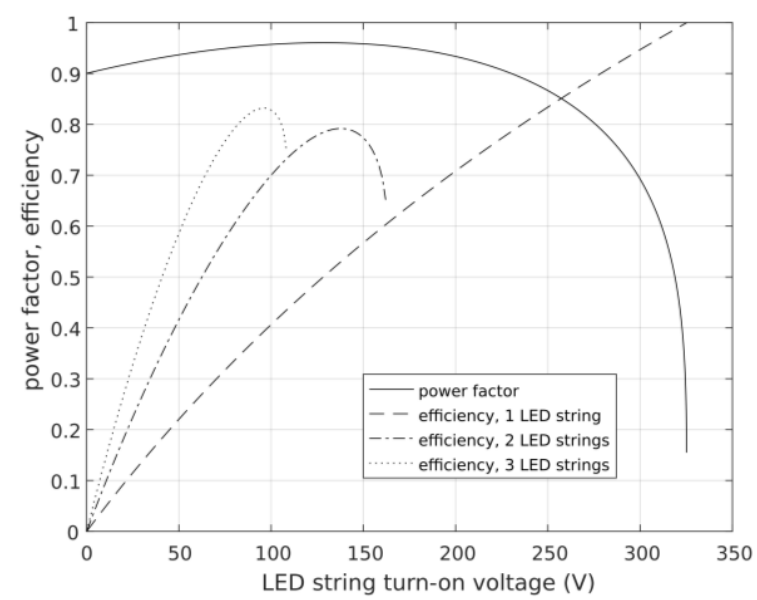

Fig. 3. Power factor and efficiency of a lamp with one, two or three identical LED strings as a function of the string conduction voltage. 
The independent variable $t_{1}$ has been replaced with the more convenient for practical use conduction (turn-on) voltage of a string $V_{L E D}$ according to the formula

$$
V_{L E D}=V_{m} \sin \frac{2 \pi}{T} t_{1}
$$

In this case the power factor reaches its maximum value $P F_{\text {max }}=0.961$ when we use a string with the turn-on voltage $V_{L E D}=128 \mathrm{~V} \quad\left(t_{l}=1.29 \mathrm{~ms}\right)$ and the efficiency becomes $50 \%$ then. It is also seen from Fig. 3 that for any value of the single LED string voltage up to about $230 \mathrm{~V}$ the power factor is maintained above 0.9 and for this upper limit the efficiency reaches $78.5 \%$.

For a lamp with two identical strings and the same currents $I_{1}=I_{2}=I$ in phases starting at $t_{1}$ and $t_{2}$ (Fig. 2) (1) is still valid and the following relationships apply:

$$
\begin{gathered}
\eta=2 \pi\left(\frac{1}{2}-\frac{t_{1}+t_{2}}{T}\right) \tan \frac{2 \pi}{T} t_{1}, \\
2 V_{L E D}=V_{m} \sin \frac{2 \pi}{T} t_{2} .
\end{gathered}
$$

The efficiency (4) as a function of conduction voltage for both LED strings is also shown in Fig. 3.

In this case the efficiency of a driver with a lamp including two $138 \mathrm{~V}\left(t_{l}=1.39 \mathrm{~ms}\right)$ LED strings reaches $79.2 \%$ and $P F$ is about 0.94 then, which is close to its maximum possible value of 0.961 .

For a lamp with three identical strings and the same currents $I_{1}=I_{2}=I_{3}=I$ in phases starting at $t_{1}, t_{2}$ and $t_{3}$ (Fig. 2) the following formulae apply together with (3) and (5):

$$
\begin{gathered}
\eta=2 \pi\left(\frac{3}{4}-\frac{t_{1}+t_{2}+t_{3}}{T}\right) \tan \frac{2 \pi}{T} t_{1}, \\
3 V_{L E D}=V_{m} \sin \frac{2 \pi}{T} t_{3} .
\end{gathered}
$$

The plot of (6) is also shown in Fig. 3. The use of three identical strings with $V_{L E D}=96 \mathrm{~V}\left(t_{l}=0.955 \mathrm{~ms}\right)$ increases the maximum available efficiency of the lamp together with the driver to $83.2 \%$ and $P F=0.956$.

In the above analyses, concerning lamps with two or three LED strings, we assumed the same turn-on voltage of all strings. However, a question arises whether we can improve electrical parameters of a lamp by using LED strings with different turn-on voltages. To examine this problem, we considered a lamp with two strings for which the following relationship has been obtained

$$
\begin{gathered}
\eta=\frac{2 \pi}{T} \times \\
\times \frac{\left(\frac{T}{4}-t_{1}\right) \sin \frac{2 \pi}{T} t_{1}+\left(\frac{T}{4}-t_{2}\right)\left(\sin \frac{2 \pi}{T} t_{2}-\sin \frac{2 \pi}{T} t_{1}\right)}{\cos \frac{2 \pi}{T} t_{1}},
\end{gathered}
$$

where:

$$
\begin{gathered}
V_{L E D 1}=V_{m} \sin \frac{2 \pi}{T} t_{1}, \\
V_{L E D 2}=V_{m} \sin \frac{2 \pi}{T} t_{2}-V_{L E D 1} .
\end{gathered}
$$

Because (1) still applies for this case, we used two values of the turn-on voltage for the first string, namely $V_{L E D I}=128 \mathrm{~V}$, for which $P F$ reaches its maximum value, and $V_{L E D I}=230 \mathrm{~V}$, for which $P F$ falls to 0.9 . The results of calculations for these two voltages are presented in Fig. 4. For the first value the efficiency reaches $78.4 \%$ when the conduction voltage of the second string is $130 \mathrm{~V}$, whereas with $V_{L E D I}=230 \mathrm{~V}$ the efficiency rises to $90.8 \%$ by choosing the second string voltage to be equal to $63 \mathrm{~V}$.

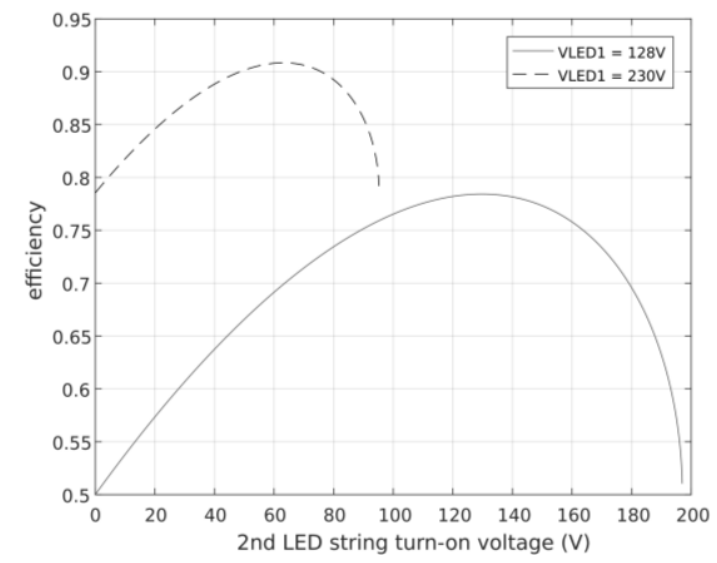

Fig. 4. Efficiency of a lamp as a function of the conduction voltage of the 2nd LED string for two values of the 1st string voltage.

As mentioned earlier, in most described designs the current is formed as a staircase waveform (Fig. 2). If we consider the simplest case of a lamp with two identical LED strings and the driver forces $I_{2}$ to be twice $I_{1}$, we get:

$$
\begin{gathered}
P F=2 \sqrt{2} \frac{\cos \frac{2 \pi}{T} t_{1}+\cos \frac{2 \pi}{T} t_{2}}{\pi \sqrt{4 \frac{t_{2}-t_{1}}{T}+16\left(\frac{1}{4}-\frac{t_{2}}{T}\right)}}, \\
\eta=\frac{2 \pi}{T} \frac{\left(t_{2}-t_{1}\right) \sin \frac{2 \pi}{T} t_{1}+2\left(\frac{T}{4}-t_{2}\right) \sin \frac{2 \pi}{T} t_{2}}{\cos \frac{2 \pi}{T} t_{1}+\cos \frac{2 \pi}{T} t_{2}},
\end{gathered}
$$

where the moments $t_{1}$ and $t_{2}$ are described by (3) and (5).

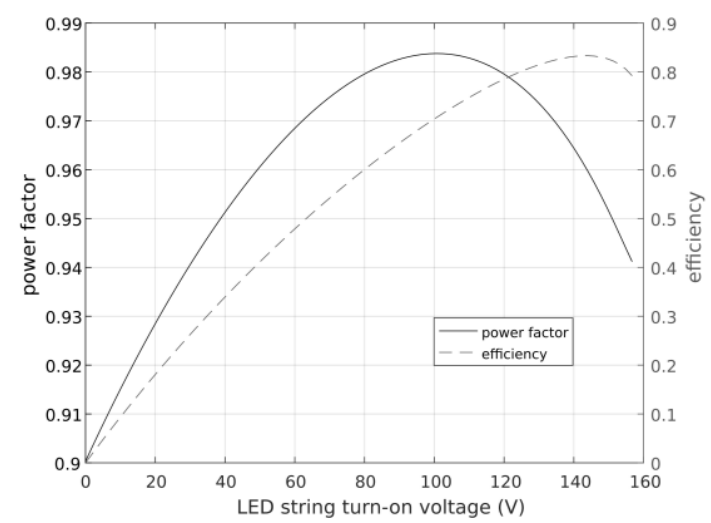

Fig. 5. Power factor and efficiency of a lamp as a function of the conduction voltage for lamps with two identical LED strings and a staircase current waveform $\left(I_{2}=2 I_{1}\right)$. 
Numerical analysis of (11) and (12) (Fig. 5) has shown that the maximum efficiency of $83.3 \%$ is obtained with $V_{L E D}=143.6 \mathrm{~V} \quad(P F=0.96)$, whereas the maximum $P F=0.984$ is obtained with $V_{L E D}=100.5 \mathrm{~V}(\eta \approx 70.6 \%)$.

\section{FLICKER}

As mentioned at the beginning of this paper, an inherent drawback of LED lamps fed from AC direct drivers, without the use of electrolytic capacitors, is large flicker at a double frequency of the mains. Despite that, most references concentrate on maximizing electrical parameters of the lamp together with its driver $(P F, \eta)$ rather than on luminous characteristics of the lamp. There are few papers only, e.g. [8], that tackle the problem of flicker and methods of its reduction. In [11] results of flicker measurement for various LED lamps supplied from different drivers are presented and compared with flicker for incandescent lamps and FCLs.

It must be noticed that by shaping the supply current as a staircase waveform, in order to make it resemble a sine wave and thus improve the power factor, the average luminous flux of a lamp is consequently reduced comparing with a lamp in which the current is the same in all phases. With three strings, for example, the current flowing through the LED strings may reach the nominal value only at $t_{3}$ (Fig. 2). At $t_{1}$ and $t_{2}$ it must obviously be smaller, whereas with the same current in all phases it may maintain the nominal value starting already from $t_{1}$. So, if the steps are equal, the currents at moments $t_{1}, t_{2}$ and $t_{3}$ will be in proportion 1:2:3. In consequence, a lamp consisting of identical strings, with the same number of LEDs, will produce luminous flux whose values at the corresponding moments will be roughly in proportion 1:4:9. This means generation of light in the form of short pulses with high intensity. With equal currents in all phases the luminous flux waveform will follow a staircase with equal steps.

The problem of flicker in LED lamps fed from AC direct drivers is certainly worth of further investigation.

\section{CONCLUSIONS}

In the literature there are many descriptions of multistring LED lamps with complicated integrated circuit drivers that achieve excellent electrical parameters, namely the power factor above 0.99 and efficiency exceeding $90 \%$. However, the overall efficiency of light generation by such lamps is reduced by using milky bulbs in order to hide the internal circuitry of the lamp.

The analyses presented in this paper show that it is possible to ensure acceptable, although lower, values of the electrical parameters using lamps with only one or two strings fed by a very simple driver. A lamp with one string requires only a bridge rectifier and a readily available IC current source with no need for high-voltage MOSFET switches. Keeping the power factor above 0.9 it is possible to achieve electrical efficiency at a level of $75 \%-80 \%$. Using such a solution for lamps with the so called LED filament, which have transparent bulbs and look like conventional incandescent lamps, may allow to achieve similar overall efficiency of light generation than in lamps with much more complicated circuitry. The main task of designers of such simple structures is to choose an optimum number of LEDs in a string. This choice may be facilitated by using the results of analyses presented in this work.

\section{REFERENCES}

[1] S. Li, S-C. Tan, C-K. Lee, "A survey, classification, and critical review of light-emitting diode drivers", IEEE Trans. Power Electronics, vol. 31, pp. 1503-1515, 2016. DOI: 10.1109/TPEL.2015.2417563.

[2] Y. Boxin, L. Zhiming, W. Zhaohui, L. Guoyuan, "Design of a novel AC driver with no current glitch based on soft switching operation", in Proc. China Semiconductor Technology International Conf. (CSTIC 2017), Shanghai, China, 2017. DOI: 10.1109/CSTIC.2017.7919893.

[3] N. Ning, W. B. Chen, D. J. Yu, C. Y. Feng, C. B. Wang, "Selfadaptive load technology for multiple-string LED drivers", Electronics Letters, vol. 49, pp. 1170-1171, 2013 DOI: $10.1049 / \mathrm{el} .2013 .2093$.

[4] J. Kim, J. Lee, S. Park, "A soft self-commutating method using minimum control circuitry for multiple-string LED drivers", in Proc 2013 IEEE Int. Solid-State Circuits Conf., San Francisco, USA, 2013, pp. 376-77. DOI: 10.1109/ISSCC.2013.6487777.

[5] S. Fu, M. Chen, X. Lee, T. Yoshihara, "A high efficiency multichannel LED driver based on converter-free technique and load adaptive method", in Proc. Int. SoC Design Conf. (ISOCC 2014), Jeju, South Korea, 2014, pp. 42-43. DOI: 10.1109/ISOCC.2014.7087585.

[6] H. Gao et al., "An electrolytic-capacitorless and inductorless AC direct LED driver with power compensation", in Proc. IEEE 2nd Int Future Energy Conf. (IFEEC), Taipei, Taiwan, 2015. DOI: 10.1109/IFEEC.2015.7361491.

[7] Y. Yeh, M. Chen, X. Li, H. Shinohara, T. Yoshihara, "AC direct multiple-string LED driver with low THD and minimum components", in Proc. Int. SoC Design Conf. (ISOCC 2015), Gyungju, South Korea, 2015, pp. 117-118. DOI 10.1109/ISOCC.2015.7401680.

[8] Y. Gao, L. Li, P. K. T. Mok, "An AC input switching-converter-free LED driver with low-frequency-flicker reduction", IEEE Journal of Solid-State Circuits, vol. 52, pp. 1424-1434, 2017. DOI: 10.1109/JSSC.2017.2656147.

[9] ENERGY STAR Program Requirements. Product Specifications for Lamps (Light Bulbs). Eligibility Criteria. Version 2.0 (valid from January 2, 2017).

[10] COMMISSION REGULATION (EU) No 1194/2012 of 12 December 2012 implementing Directive 2009/125/EC of the European Parliament and of the Council with regard to eco-design requirements for directional lamps, light emitting diode lamps and related equipment.

[11] J. Checinski, Z. Filus, "Stability of the luminous flux of LED lamps supplied from the 230VAC network", Przeglad Elektrotechniczny, vol. 94, pp. 5-8, 2018. (in Polish). DOI: 10.15199/48.2018.08.02. 